\title{
EDITORIAL
}

\section{Children are key to CVD prevention}

$\mathrm{n}$ adults, the increased cardiovascular risk associated with factors such as high blood pressure, elevated lipid levels, smoking, a sedentary lifestyle, and being overweight is well known; however, it is important to recognize that these risk factors often develop and begin to detrimentally affect health in childhood.

Unhealthy behavioral habits begin at a very early age. Lack of exercise and a poor diet lead to excess weight and obesity, which in turn contribute to raised blood pressure, glucose intolerance, and hyperlipidemia. The WHO currently estimates that, worldwide, more than 42 million children under the age of 5 years are overweight [http:// www.who.int/dietphysicalactivity/childhood/en/], predisposing them to the early onset of cardiovascular conditions. The Global Youth Tobacco Survey reported that more than $10 \%$ of adolescents currently use tobacco products, with almost $25 \%$ of these children lighting their first cigarette before the age of 10 years [http://www.cdc.gov/ tobacco/global/gyts/index.htm]. Several reports from the Bogalusa Heart Study show that pathophysiological changes in the cardiovascular system, such as the development of fatty streaks and early atherosclerotic lesions, occur in children as young as 5 years of age and that these changes are correlated with the presence of risk factors [http://www.som.tulane.edu/cardiohealth/ bog.html]. Clearly, the primary prevention of cardiovascular disease (CVD) should extend beyond risk factor reduction in adults. Promoting heart-healthy behaviors in young children is also essential to achieving this goal and to reducing the global burden of CVD.

The early years of life, when behaviors are still being learnt, is an excellent window of opportunity to educate children about cardiovascular health. Teaching healthy habits to children is far easier than changing wellestablished unhealthy behaviors in adults. There are two important aspects to this educational message. Firstly, children need to learn heart-healthy behaviors at a young age to ensure good health during childhood and to prevent the early onset of cardiovascular disease. Secondly, these behaviors should be continued into adulthood and throughout life to maintain a healthy way of living. It follows that children who recognize the importance of a balanced diet and physical exercise, and the harmful effects of smoking are likely to carry these values with them for the rest of their lives and, eventually, pass them on to their own offspring.

Children also have an important role in educating their parents and siblings about healthy living. The FleurbaixLaventie-Ville-Santé Study showed that a school-based program to improve nutritional knowledge in children aged 6-12 years also had a beneficial impact on the

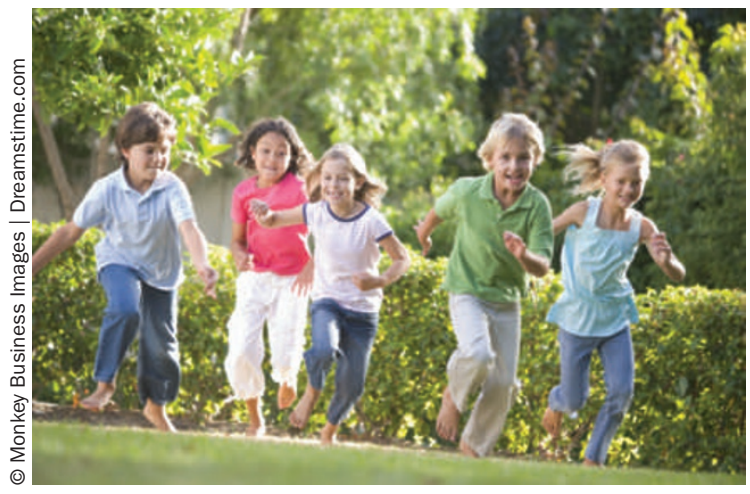

dietary habits of their families [Romon, M. et al. Public Health Nutr. 12, 1735-1742; 2009]. In addition, the World Heart Federation (WHF) seeks to empower young people by supporting the Youth for Health (Y4H) campaign, a youth advocacy program that allows children to mentor and educate their peers about the importance of preventing cardiovascular risk factors. This 'by children for children' approach gives them a sense of ownership and recognizes the importance of leading by example.

There are many ways in which we can encourage young children to adopt healthy behaviors, and several national and international initiatives are already underway. Importantly, strategies should be age-appropriate, applicable across social, cultural, and educational groups, and, ideally, be fun and engaging for the child. Such programs can be conducted through pediatric health clinics, school systems, the local community, and the media.

One of the most far-reaching of these projects is being conducted by the Sesame Workshop, which is the largest informal educator of children in the world, with viewers in 120 countries. The Healthy Habits for Life initiative [http://www.sesameworkshop.org/initiatives/health/ healthyhabits] was launched in 2004 to promote enjoyable healthy eating and exercise in children and their parents through the use of DVDs, storybooks, coloring kits, and activity and recipe cards featuring the characters from Sesame Street. A bilingual, outreach extension of this program is also being initiated to target low-income families and highlight that a lack of financial resources need not be a barrier to a healthy lifestyle. Children respond to this project because it is fun and they identify with the characters.

In a different initiative, the WHF has produced the Eat for Goals book for children, which uses international footballers as role models to encourage healthy eating and participation in sports. This resource recognizes the way in which children admire and want to imitate their heroes.

\author{
4 ... patho- \\ physiological \\ changes \\ in the \\ cardiovascular \\ system... \\ occur in \\ children \\ as young \\ as 5 years \\ of age... 77
}

Alexandra King is

Senior Editor of Nature

Reviews Cardiology.

Valentin Fuster is

Editor-in-Chief of Nature Reviews Cardiology.

Competing interests The authors declare no competing interests. 
4 Promoting heart-healthy

behaviors

in young children is ... essential 77
Launched in February 2010 by US First Lady Michelle Obama, the Let's Move initiative [http://www.letsmove.gov/] is another high-profile campaign, which has the aim of eliminating the epidemic of childhood obesity within a generation. Access to affordable, healthy food for all, improving the nutritional value of school meals, and encouraging children to be physically active for an hour every day are the cornerstones of this program. As with the Sesame Workshop, Let's Move utilizes games, videos, activities, and visual aids for children to make healthy living fun. The 'neighborhood' approach adopted in Let's Move-involving children, parents, teachers, health-care providers, and community-leaders-reinforces the idea of doing something together; children can participate in activities with their friends, which is enjoyable and likely to improve adherence to lifestyle changes.

Similar projects are being conducted elsewhere; in Australia, for example, the Go for 2 and 5 initiative uses recipes, activities, and educational challenges to help children understand the importance of eating their recommended two servings of fruit and five servings of vegetables each day. The Get Moving campaign targeted children aged 5-17 years to increase their levels of physical activity. A report on Get Moving, published in January 2007, described encouraging results with a significant decrease in mean weekend sedentary time in children aged 9-12 years, from $5.9 \mathrm{~h}$ before the start of the campaign to $4.5 \mathrm{~h}$ at the 3 -month follow-up [http:// www.health.gov.au/internet/healthyactive/publishing.nsf/ Content/CD99B2A715DB9214CA2572DC001855A8/ \$File/getmoving-eval-jul07.pdf].

The cardiovascular health of future generations depends on the health behaviors we adopt today. Reducing the global burden of obesity, hypertension, diabetes, and hyperlipidemia by preventing the onset of these conditions in young children is vitally important. Future initiatives should build on the strengths of the existing projects highlighted above, while recognizing the potential for children themselves to serve as influential advocates for healthy lifestyles. We have a wonderful opportunity to prevent CVD by educating the next generation of adults, while they are still children, to adopt and maintain healthy behaviors throughout life and eliminate the unhealthy habits of the past.

doi:10.1038/nrcardio.2010.66 